\title{
Procédés et opérations des SuJets Parlants Chez F. De SAussure
}

\author{
Pierre-Yves Testenoire \\ ÉSPÉ de Paris, Sorbonne Université, UMR 7597 Histoire des théories linguistiques, \\ Paris, France
}

\section{Résumé}

La rupture proclamée par Saussure avec l'approche psychologique du langage, relayée par sa lecture structuraliste, ne doit pas dissimuler l'importance accordée dans sa théorisation à l'activité cognitive des locuteurs. En effet, la critique de la psychologie chez Saussure va de pair avec une définition de la langue en synchronie par la conscience des sujets parlants. C'est dans ce cadre que sont développées, dans les cours de linguistique générale, les notions de "procédé» et d'«opération des sujets parlants» sur lesquelles porte cet article. On s'intéressera à la carrière de ces notions mobilisées pour l'analyse de faits morphologiques et syntaxiques et spécifiquement la construction de ce que Saussure appelle les «entités abstraites».

\section{Mots-clés}

Psychologie, cognition, sujet parlant, conscience de la langue, sentiment de la langue

\section{Abstract}

The rupture proclaimed by Saussure with the psychological approach of language, conveyed by his structuralist view, must not conceal the importance of the speaker's cognitive activity, which is given in his theories. Indeed, Saussure's criticism of psychology goes hand in hand with the definition of language in synchrony through the consciousness of the speakers. This is the context in which the notions of "process" and "operation of speakers" are developed, in general linguistic courses, which are the main focus of this paper. This article will address the career of these notions, used for the analysis of morphological and syntactic facts, and in particular the construction of what Saussure calls "abstract entities".

\section{Keywords}

Psychology, cognition, speaker, language consciousness, linguistic feeling

\section{OPÉRATIONS SANS REPRÉSENTATION}

Les deux termes du couple conceptuel que se propose d'interroger ce numéro -opération et représentation - n'occupent pas la même place dans la pensée théorique de F. de Saussure. Autant la notion d'opération y joue un rôle réel, bien que discret, autant celle de représentation lui paraît étrangère. La démarche saussurienne peut même être comprise comme un effort pour affranchir la théorie du langage du concept de représentation. Pour Saussure, non seulement, la langue ne représente ni le monde ni la pensée - une position anti-référentialiste et antireprésentationiste affirmée à maintes reprises - mais le processus de représentation 
est également exclu de son fonctionnement sémiotique car, dans la conception saussurienne du signe, le signifié ne représente pas le signifiant, pas plus que le signifiant ne représente le signifié. Les deux faces du signe linguistique sont conçues comme une « dualité », une « association »-vocabulaire que le linguiste reprend à la psychologie associationiste mais dont il se démarque (v. Bergounioux 1995). Signifiant et signifié ne préexistent pas à leur association en signe linguistique : l'un ne s'origine pas dans autre. Leur rapportn'est, dès lors, ni hiérarchisé, ni prédeterminé, ni orienté : une position qui tranche avec la conception traditionnelle de la représentation de l'idée par le signe ou du sens par le son. Le seul domaine pour lequel Saussure conserve, en définitive, le concept de «représentation» est celui du rapport entre langue et écriture, comme en témoigne le chapitre 6 de l'introduction du Cours de linguistique générale intitulé «Représentation de la langue par l'écriture». Cependant, l'absence de définition claire de ce rapport de représentation et les variations qui s'observent entre les cahiers d'étudiants ayant servi de source à ce chapitre laissent ouverte une pluralité d'interprétations sur la conception saussurienne du rapport entre langue et écriture (v. Chiss \& Puech 1983 et Testenoire 2017).

La notion d'opération, quant à elle, joue un rôle dans l'appareil théorique saussurien, spécifiquement dans la façon dont le linguiste envisage l'articulation du langage et de la pensée. Sur cette question, la théorisation saussurienne articule deux positions qui peuvent passer pour contradictoires ou, tout du moins, qui semblent entrer en tension.

a) La première est la critique constante, chez Saussure, de la psychologie du langage. L'arrière-fond de cette critique est la problématique, propre au dernier tiers $\mathrm{du} \mathrm{XIX}^{\mathrm{e}}$ siècle, de l'autonomisation, à fois épistémologique et institutionnelle, de la linguistique. Cette stratégie d'autonomisation se traduit chez Saussure par un effort de définition d'un objet qui soit spécifique à la science linguistique et qui légitime son point de vue par rapport au modèle dominant d'alors: la psychologie. Les divers développements que Saussure consacre à la classification des sciences récusent le simple emboitement de la linguistique dans la psychologie que proposent les collègues dont il discute les thèses-Wundt (1900), Naville (1901), Sechehaye (1908) Bally (1909) ${ }^{1}$. Saussure ne conçoit une inclusion de la linguistique dans la psychologie

1 Ces questions de classification des sciences et le lien entre linguistique et psychologie sont particulièrement discutés dans le compte rendu que Saussure fait du livre de Sechehaye, Programme et méthodes de la linguistique théorique (CLG/E 3330= Saussure 2002, p. 258261), dans la discussion des premiers travaux de Charles Bally (CLG/E 3347 = Saussure 2002, p. 272-273 et Amacker 1994b, p. 132) et dans l'introduction de chacun des trois cours de linguistique générale. 
que comme une conversion à la linguistique de la psychologie lorsque celle-ci «s'apercevra que la langue n'est non pas une de ses branches, mais l'ABC de sa propre activité $»^{2}$. L'articulation entre linguistique et la psychologie sociale est finalement théorisée, à partir des années 1900, par la science sémiologique dont l'objet linguistique fournira le patron. De cet effort pour déterminer la spécificité de la linguistique et de son objet découle, dans les textes saussuriens, une critique permanente de l'approche psychologique du langage: Saussure n'a de cesse d'excommunier de la science du langage telle qu'il la conçoit toute tentative de description d'une activité cognitive qui ne se manifesterait pas par une activité langagière. Face au domaine de la physiologie qui ne s'intéresse qu'au signe sans idée, ce que Saussure appelle dans le manuscrit dit de l'Essence double (1891) «la figure vocale», et face à celui de la psychologie qui ne s'intéresse qu'à l'idée sans signe, Saussure fait de la linguistique le domaine des articulations, ce qu'il appelle « la forme-sens » dans l'Essence double et qu'il appellera le «signe linguistique » dans les cours de linguistique générale.

b) Cette mise à distance de la psychologie n'empêche pas l'appareil saussurien d'incorporer, de façon diffuse, plusieurs notions psychologiques dans lesquelles se reconnaissent aussi bien l'empreinte de l'herbartisme, qui fournit le soubassement psychologique aux théories néo-grammairiennes (Samain 2016), que l'influence plausible de la psychologie expérimentale de tradition française-Taine, Ribot, Janet (De Palo [2001] 2016) ${ }^{3}$. Aussi Saussure reconnaît-il que «tout est psychologique dans la linguistique ${ }^{4}$. Sa critique de l'approche psychologique va de pair avec une définition de la langue en synchronie par la conscience des sujets parlants. Cet aspect, qui a été minoré par la lecture immanentiste développée par le structuralisme, a été redécouvert à la faveur de la relecture des textes effectuée dans la foulée de la découverte et la publication de nouveaux manuscrits au tournant du $\mathrm{xx}^{\mathrm{e}}$ et $\mathrm{xxI}^{\mathrm{e}}$ siècles. Les formulations des textes saussuriens sont, sur ce point, explicites et constantes à

2 La formulation figure dans une des notes Item écrites entre 1898 et 1903 : «Item. Pour aborder sainement la linguistique, il faut l'aborder du dehors, mais non sans quelque expérience des phénomènes prestigieux du dedans. Un linguiste qui n'est que linguiste est dans l'impossibilité à ce que je crois de trouver la voie permettant de classer les faits. Peu à peu la psychologie prendra pratiquement la charge de notre science, parce qu'elle s'apercevra que la langue n'est non pas une de ses branches, mais l'ABC de sa propre activité. » (CLG/E 3315.3 = Saussure 2002, p. 109).

3 Sur l'influence de la littérature psychologique sur la théorie saussurienne, v. également Amacker 1994a et Bergounioux 1995. L'identification des sources précises de la pensée saussurienne se heurte à une difficulté identifiée depuis le travail de Koerner (1971) : l'habitude qu'a Saussure de ne pas mentionner ses lectures est accentuée en ce qui concerne ses influences non linguistiques (philosophiques, psychologiques, dans les sciences sociales, économiques ou naturelles).

4 La formule est consignée par Marguerite Sechehaye lors du troisième cours (CLG/E 111 III S 1.2). 
travers les années. Ainsi, dès l'Essence double, Saussure s'interroge sur les points de vue possibles qui donnent consistance aux faits de langage et il pose qu' «il n'existe linguistiquement que ce qui est aperçu par la conscience, c'est-àdire ce qui est ou devient signe» (Saussure 2002, p. 45 = Saussure 2011, p. 103). Cette définition de la langue en synchronie par la conscience des sujets parlants se retrouve une vingtaine d'années plus tard dans l'enseignement de linguistique générale, aussi bien dans le deuxième cours (1908-1909):

Dans l'ordre synchronique il n'y a qu'une variété et qu'une méthode possible [...] : observer ce qui est ressenti par les sujets parlants. (CLG/E, 1503-1504 II R 85)

que dans la définition de la linguistique statique donnée dans le troisième cours (1910-1911):

La linguistique statique s'occupera de rapports logiques et psychologiques $<$ entre termes $>$ coexistants < tels qu'ils sont $>$ aperçus par la même conscience collective (dont du reste une conscience individuelle peut donner l'image - chacun de nous a en soi la langue) et formant un système. (CLG/E, 1660 III C 362)

L'analyse morphologique - qui est pour Saussure, comme pour tout comparatiste $\mathrm{du} \mathrm{XIX}^{\mathrm{e}}$ siècle, le noyau dur de la description linguistique - ne peut s'opérer que si on adopte le point de vue synchronique, c'est-à-dire celui des sujets parlants. En dehors de ce point de vue, on tombe là encore soit dans le substantialisme de la phonétique historique soit dans la «métaphysique» de la psychologie du langage. Le primat accordé à la conscience des locuteurs et à ses variations d'époque - « subconscience», «demi-conscience», «inconscience ${ }^{5}$ - rentre en conflit direct avec la doxa d'il y a cinquante ans qui faisait de la linguistique saussurienne une linguistique de l'exclusion du sujet.

Critique de la psychologie et réhabilitation de la signification et conséquemment de la conscience des sujets parlants délimitent donc la zone dans laquelle la théorisation saussurienne examine les opérations mentales attribuables aux locuteurs dans leurs activités langagières. C'est dans ce cadre que sont développées les notions de «procédés» et d'«opérations des sujets parlants » sur lesquelles nous proposons de nous pencher. Nous nous concentrerons pour cela sur un moment de la pensée saussurienne : celui reflété par les cours de linguistique générale que Saussure dispense à la fin de sa carrière. Nous nous appuierons prioritairement, à cet effet, sur les notes

5 Si toutes ces expressions sont attestées, on sait qu'en revanche inconscient n'apparaît pas sous sa forme nominale dans le corpus saussurien. Sur la conception relative de la conscience chez Saussure et sur ce qui la différencie de l'inconscient freudien, v. Arrivé [2012] 2016. 
consignées par ses étudiants ${ }^{6}$. L'ambition de décrire, sur cette question, un moment homogène de la pensée saussurienne ne nous interdira pas quelques excursions dans les écrits théoriques antérieurs du linguiste mais en rendant compte à chaque fois de la chronologie des textes - précaution méthodologique trop peu souvent observée mais, à notre sens, indispensable pour qui s'intéresse à l'historicité de sa pensée.

\section{Conscience et Sentiment de la langue}

Les trois cours de linguistique générale que Saussure dispense à l'Université de Genève - le premier de janvier à juillet 1907, le second pendant l'année universitaire 1908-1909, le troisième pendant l'année universitaire 1910-1911 - ne sont pas homogènes. Chacun présente un contenu et un plan qui différent des deux autres et que le professeur organise en prenant en compte les divers paramètres liés à la situation d'enseignement: temps imparti, programme annoncé, nombre et niveaux des auditeurs... Le premier cours occupe, de ce point de vue, une place particulière. Saussure récupère cet enseignement à l'automne 1907 en raison du départ précipité d'un collègue alors que le semestre est déjà commencé et que les étudiants sont déjà inscrits ${ }^{7}$. Le programme du cours de «linguistique générale» que le professeur élabore doit prendre en compte l'ancien programme du cours de «linguistique» sur la base duquel les étudiants se sont inscrits. Adressé à des étudiants non spécialisés en langues anciennes, le premier cours s'avère surtout propédeutique et négatif: son objet est de dissiper les idées reçues et les erreurs méthodologiques de la linguistique de son temps. Aussi des trois cours il est celui qui a fourni le moins de concepts ou de formulations qui ont fait le succès du $C L G$, même si certains thèmes - la linéarité, les distinctions synchronie/diachronie, langue/parole - y apparaissent déjà ${ }^{8}$. Mais le premier cours est aussi, des trois, le

6 Les cahiers d'étudiants aux trois cours de linguistique générale sont cités d'après l'édition critique du Cours de linguistique générale de Rudolf Engler (Saussure 1968). Les manuscrits de linguistique générale découverts dans les années 50 sont cités selon la numérotation de l'édition de Rudolf Engler (Saussure 1974) et on indique aussi la pagination de l'édition de Simon Bouquet et de Rudolf Engler (Saussure 2002) dans laquelle ces textes ont été repris (section Anciens documents). Enfin, les manuscrits de linguistique générale découverts en 1996, dont le projet d'ouvrage De l'essence double du langage, sont cités d'après les deux éditions existantes : celle de Simon Bouquet et de Rudolf Engler (Saussure 2002) et celle de René Amacker (Saussure 2011). On renvoie aux deux paginations.

7 Sur ce remplacement et les circonstances administratives et pédagogiques du premier cours v. Joseph (2012, p. 489-493), Vincent (2013) et Chidichimo (2017).

8 Le premier cours a surtout été utilisé par Bally et Sechehaye pour la rédaction de la troisième partie du Cours de linguistique générale, «Linguistique diachronique» (v. Godel 1957, p. 103112), soit la partie la moins lue et la moins commentée. Comme l'écrit De Mauro: «Dans le plan ultime de Saussure l'analyse des phénomènes historico-évolutifs devait non pas suivre mais précéder la présentation de la langue. Indubitablement, si les éditeurs avaient suivi cette voie, les troisième, quatrième et cinquième parties du CLG auraient été considérées avec plus d'attention, et la vue historicisante de Saussure de la langue élaborée par Saussure aurait apparu plus clairement au lecteur.» (Saussure [1916] 1967, p. 472). 
plus empirique, celui où Saussure se penche concrètement sur les procédures d'analyse des linguistes mais aussi des locuteurs. C'est la raison pour laquelle il accorde une importance toute particulière aux notions de «conscience de la langue» et de «sentiment de la langue».

Les données quantitatives donneront une première idée de l'importance accordée à ce thème: les cahiers de notes de Riedlinger prises au premier cours - principale source conservée pour ce cours- comptent 33 occurrences de «conscience» et 18 mentions de la notion de sentiment linguistique. En comparaison, les cahiers de Riedlinger du deuxième cours et ceux de Constantin du troisième cours, pourtant plus longs, présentent respectivement 7 et 13 occurrences du terme «conscience», et 3 et 2 occurrences de «sentiments». Ce contraste n'indique pas uniquement la dépsychologisation des deux derniers cours, davantage centrés sur la description interne du système linguistique, il traduit aussi le travail de décantage terminologique opéré dans le dernier cours.

Les notions de «sentiment» et de «conscience de la langue», si elles ne paraissent pas équivalentes, ne sont pas toujours discriminées dans l'enseignement saussurien. La notion de sentiment linguistique, qui se rencontre çà et là dans les textes antérieurs de Saussure ${ }^{9}$, traverse également la littérature linguistique du XIX ${ }^{\mathrm{e}}$ siècle sans recevoir de définition précise ${ }^{10}$. Dans le premier cours, le sentiment linguistique des locuteurs porte sur des éléments précis de l'analyse morphologique : les sujets parlants ont tour à tour le sentiment "d'un préfixe ${ }^{11}$, «d'un radical $»^{12}$, «de la racine $»^{13}$, «d'alternances» morphologiques ${ }^{14}$, ou plus vaguement «d'une unité ${ }^{15}$ ou d'un «élément» linguistiques ${ }^{16}$. L'imprécision du terme permet de réhabiliter, dans le sillage des néogrammairiens, le rôle des locuteurs dans les mécanismes de transformation des langues sans déterminer le degré de conscience auquel se situe cette intervention. En plusieurs endroits, cependant, «sentiment de la langue» est employé de façon équivalente à «conscience», soit que les deux termes soient apposés de façon synonymique ${ }^{17}$, soient qu'ils se relaient indifféremment ${ }^{18}$.

\section{V. Chidichimo 2009 et Fadda 2013.}

10 On la trouve dans la tradition française chez Nodier, Littré, Bréal et également, en allemand («Sprachgefühl») chez Schleicher, Gabelentz ou Paul, comme l'a montré G. Siouffi lors d'un atelier consacré au sentiment linguistique chez Saussure organisé dans le cadre du Colloque international Le Cours de linguistique générale 1916-2016 qui s'est tenu à Genève en janvier 2017.

11 CLG/E 2100 I R 2.40 et 2590 I R 2.37.

12 CLG/E 2556 I R 2.49 et CLG/E 2506 I R 2.72.

13 CLG/E 2449 I R 2.56.

14 CLG/E 2544 I R 2.93.

15 CLG/E 2807 I R 2.51.

16 CLG/E 2526 I. R 2.89.

17 CLG/E 2556 I R 2.49, CLG/E 2527 II R 103.

18 CLG/E 2526 I R 2.92 et CLG/E 1802 II R44. 
De fait, la distinction entre le sentiment et la conscience linguistiques ne correspond pas, comme on pourrait le croire, à une opposition entre une perception de niveau faible et de niveau élevé des faits de langue, la conscience étant toujours relative pour Saussure ${ }^{19}$. L'opposition se situe à un autre niveau dans les emplois saussuriens : «sentiment de la langue» fait davantage intervenir la subjectivité et l'activité des locuteurs - elle est utilisée pour la description des phénomènes d'analogie - «conscience» est plus passive, objective. C'est vraisemblablement l'imprécision du terme « sentiment» qui conduit Saussure à l'éliminer du troisième cours et à ne conserver que «conscience de la langue $»^{20}$.

Les deux notions, en dépit de leurs nuances, présentent des caractéristiques communes. L'une d'entre elles est leur nature sociale: un sentiment ou une conscience linguistique est attribué à un sujet en tant qu'il est le reflet du sentiment ou de la conscience linguistique d'une collectivité. Un autre point commun réside dans le caractère agentiel que revêt la langue dans ces notions de sentiment et de conscience linguistiques telles que Saussure les emploie. Plusieurs commentateurs (Chidichimo 2009; Fadda 2013; Arrivé 2016) ont relevé que les syntagmes «conscience de la langue » et « sentiment de la langue » avaient, dans les cours de linguistique générale, deux significations selon les emplois, le complément du nom «de la langue» recevant tantôt une acception objective tantôt une acception subjective. Or, la répartition de ces emplois obéit à une progression chronologique. Dans les deuxième et troisième cours, en effet, ne sont attestés que des emplois avec l'interprétation objective, selon laquelle ce sont les sujets qui ont conscience ou sentiment de la langue. À titre d'exemple :

aimer: aimable $=$ entamer: $\mathrm{x}$ Cette formule revient à ceci, c'est que $<$ la conscience intérieure, $>$ le sentiment de la langue, en tirera ceci: je puis remplacer -er par -able. (CLG/E 2527 II R 103)

Tout peuple prend le mieux conscience de la langue, même placé assez bas dans l'échelle de la civilisation. (CLG/E 2848 III C 21)

19 Preuve de ce relativisme, Saussure parle, dans ses cours, aussi bien de «vague conscience» (CLG/E 2100 I R 2.40), de «conscience latente» (CLG/E 2064 II R 96) que de "volonté inconsciente » (CLG/E 1434 III C 348).

20 Les deux seules occurrences de «sentiment» dans le troisième cours concernent le «sentiment que nous avons de l'arbitraire » (CLG/E 1178 IIII C 312) et le « sentiment actuel» des locuteurs pour qui les faits évolutifs n'ont pas d'existence (CLG/E 1606 IIII C 364), soit un emploi très différent du «sentiment» des éléments de l'analyse linguistique présent dans le premier cours. L'élimination de «sentiment» en 1910-1911 n'est pas un hasard mais correspond au travail de systématisation terminologique effectué pour ce cours qui condamne certains flottements terminologiques : à titre d'exemple, la notion de «discours » employée dans les premiers et deuxième cours est évacuée dans le troisième en raison de la redéfinition du concept de parole (v. Testenoire 2016). 
Et c'est exclusivement dans le premier cours qu'on rencontre, à côté de cette interprétation la plus spontanée de ces syntagmes, des acceptions subjectives où la langue se trouve dotée de sentiment ou de conscience:

Un second fait, c'est que la langue a la conscience non seulement des éléments mais aussi de $<$ l'influence $>$ qu'ils exercent les uns sur les autres quand on les place dans un certain ordre; la langue a le sentiment de leur sens logique, de leur ordre. La syntaxe intérieure du mot est abstraite de la comparaison des formes aussi bien que les éléments <eux-mêmes>. (CLG/E 2538 I R 2.92)

La langue ne connaît pas les noms de radical, suffixe, etc. mais on ne peut lui refuser la conscience et l'utilisation de ces différences. La formation analogique est la vérification de cette analyse de la langue, mais il faut se rendre compte de la différence de fonction de toutes ces unités, dont les unes sont plus ou moins présentes, d'autres tout à fait présentes à la conscience de la langue. (CLG/E 2180 I R 2.32)

Nous avons là l'exemple d'une langue qui, à un certain moment a poussé très loin la conscience, le sentiment d'un radical. » (CLG/E 2556 I R 2.49)

Dans chacun de ces extraits, le sujet « la langue » peut sans peine être remplacé par « le sujet parlant». D’autres formulations semblables figurent dans le premier cours, où la langue se trouve en position de sujet actif et ceux qui l'emploient dans une position passive :

La régularité de structure quelle qu'elle soit aide les sujets parlants à reconnaître l'unité radicale en développant chez eux un certain sentiment linguistique. (CLG/E2815 I R 2.52)

Cela nous amène à la question de savoir jusqu'à quel point la langue distingue les racines des suffixes, etc. (CLG/E 2829 I R 2.76)

Enfin, il y a des cas où on circule entre emplois objectifs et emplois subjectifs, passant successivement de la conscience qu'ont les sujets parlants à celle dont dispose la langue :

On peut constater que dans la généralité des cas <les sujets parlants ont conscience qu'>après avoir détaché du mot le préfixe, il reste une partie du mot qui est elle-même une unité : re-commencer. Ce n'est pas toujours le cas : séparer! C'est pour la catégorie du préfixe qu'il y a le moins de contestation sur le fait qu'il est conscient à la langue : <en observant d'autres cas, mais sans aller au-delà de sa propre conscience de la langue $>$ on voit qu'on ne peut refuser à la langue la conscience de ce que nous appelons $<$ le préfixe $>$ employé d'une certaine façon qui sera abstraite des analogies. C'est même ce qui fait du préfixe une catégorie à part. (Nous soulignons. CLG/E 2623 I R 2.43)

Ce qu'il faut retenir de ces oscillations - qui se rencontrent aussi dans les textes théoriques des années $1890^{21}$ - c'est la codétermination, dans le premier cours, entre, d'une part, la langue et, de l'autre, la conscience ou le sentiment des sujets 
parlants. Cette co-détermination aboutit, dans le premier cours, à une assimilation entre ces notions : langue, sentiment et conscience des sujets finissent par devenir, dans certains passages, interchangeables. Dans la perspective synchronique, la conscience linguistique des sujets valide ce qui relève de la langue et permet de l'observer : la méthode proposée est de « considérer comme réel ce que la conscience de la langue reconnaît, ratifie, et comme irréel ce qu'elle ne reconnaît pas » ${ }^{22}$. Reste la question de l'accès à la conscience linguistique des sujets. Saussure n'envisage, à cette fin, ni l'enquête, propre aux dialectologues, ni l'introspection, pratiquée par la psychologie mentaliste. Il fonde son approche sur l'analyse de marques morphologiques et morphosyntaxiques des énoncés linguistiques prises comme autant d'indices de l'activité mentale des sujets parlants.

\section{3 «Procédé »VS «Processus »}

Toujours dans le premier cours, Saussure pose au sein des procédures qui affectent le système linguistique, une distinction importante qui entre en résonnance avec le débat traversant la littérature linguistique d'alors sur le rôle de la conscience dans le fonctionnement et le changement linguistiques. Alors que, par exemple, Victor Henry, dans ses Antinomies linguistiques, distingue la «conscience de l'acte» de l'«inconscience des procédés» (Henry 1896, p. 64-78), Saussure fait basculer la notion de «procédé» du côté de la conscience en l'opposant au «processus». La distinction est posée dans le premier cours au sujet de l'agglutination:

Qu'est-ce que le processus agglutinatif? C'est un processus, disons-nous, et non un procédé : un procédé implique une volonté, une intention; on méconnaitrait le caractère de l'agglutination en y introduisant quelque chose de volontaire; c'est justement cette absence de volonté qui est un des caractères par lequel l'agglutination se distingue de la création analogique. (CLG/E 2677 I R 2.81)

La partition est claire et employée avec constance dans les cours de linguistique générale : elle rejoint la thèse néo-grammairienne de la complémentarité entre facteur physiologique et facteur psychologique dans le changement linguistique. Le « processus » est réservé aux opérations qui affectent la langue sans faire intervenir la conscience ou la volonté des locuteurs (le changement phonétique et l'agglutination). Inversement, le "procédé» relève d'une opération consciente et volontaire, dont relèvent l'analogie et l'étymologie populaire. Conséquemment, Saussure parle régulièrement de «processus agglutinatif» et de « procédé analogique $»^{23}$ en donnant toujours à ce terme de "procédé» le sens précis «d'opération consciente».

22 CLG/E 2163 II R 108.

23 Havet parle déjà, en 1875, de «procédé analogique» dans un texte où avant Herman Paul, il introduit la quatrième proportionnelle pour décrire l'analogie, v. Morpurgo-Davies 1978, p. 41 . 


\section{4 «PROCÉDÉS DES GRAMMAIRIENS »VS «PROCÉDÉS DES SUJETS PARLANTS »}

Au sein de ces procédés conscients, Saussure établit une seconde distinction en confrontant les analyses morphologiques des grammairiens et celles développées par les locuteurs :

$<$ Jusqu'à présent $>$ nous ne nous sommes pas occupés des analyses des grammairiens et nous n'avons recherché que ce qui est vivant dans la conscience des sujets parlants. C'est un danger, linguistique, de mêler les décompositions faites à différents points de vue avec $<$ celles $>$ faites par la langue; il est bon de faire le parallèle <et de confronter les procédés du grammairien pour décomposer le mot dans ses unités avec le procédé des sujets parlants. Par> cette opposition on pourra mieux définir jusqu'où va l'analyse intérieure et instinctive. Le procédé instinctif par lequel on décompose les mots, notamment dans les langues indo-européennes, est des plus simples et repose sur des opérations parfaitement définies quoique les linguistes ne soient pas au clair sur ce qui justifie l'analyse. (Nous soulignons. CLG/E 2781 seq. I R. 2.58-59)

Entre l'analyse savante des grammairiens et l'analyse spontanée des locuteurs, c'est à cette dernière qu'est donnée la priorité :

La langue ne peut pas procéder comme le grammairien; elle est à un autre point de vue et les mêmes éléments ne lui sont pas donnés; elle fait ce qui par le grammairien est considéré comme des erreurs, $<$ mais $>$ qui n'en sont pas car il n'y a de sanctionné par la langue que ce qui est immédiatement reconnu par elle. Entre l'analyse subjective des sujets parlants eux-mêmes (qui seule importe !) et l'analyse objective des grammairiens il n'y a donc aucune correspondance, quoiqu'elles soient fondées toutes deux en définitive sur la même méthode (confrontation des séries). $<\mathrm{Si}$ le grammairien opère subjectivement et objectivement il n'arrive pas au même résultat et l'on peut $>$ dire dans ce cas qu'une des deux analyses ne se justifie pas. Quelle est la valeur de l'analyse objective par rapport à l'autre? (CLG/E 2759-2762 I R. 2.65-66)

Avec cette distinction entre « analyse subjective des sujets parlants » et « analyse objective des grammairiens» c'est la distinction entre métalinguistique et épilinguistique qui est explicitement thématisée. En assimilant la langue à la conscience des sujets parlants, Saussure légitime toutes les manifestations de l'activité épilinguistique des locuteurs - analyses spontanées, procédures de remotivation, associations paronomastiques, calembours...- qui donnent accès aux opérations d'analyse des sujets parlants. Sur ce point, Saussure ne partage pas la position normative de ses deux disciples, Bally et Sechehaye. Cette posture normative affleure çà et là dans la rédaction du Cours de linguistique générale, notamment au sujet des associations fondées sur le seul signifiant que les éditeurs, dans une note, qualifient de « procédures anormales » qui relèvent d'une « catégorie inférieure » et qui reposent sur des «confusions absurdes ${ }^{24}$. Un tel discours 
axiologique ne se retrouve pas chez Saussure pour qui les analyses spontanées et les associations fondées sur le seul signifiant sont aussi légitimes que les autres rapports associatifs-et on sait tout l'intérêt qu'il porte aux phénomènes d'homophonie et aux jeux verbaux dans ses écrits de linguistique générale et dans sa recherche des anagrammes ${ }^{25}$. Peu importe, pour Saussure, si les procédures d'analyse sur lesquelles reposent ces jeux de langage coïncident ou non avec les analyses savantes. Ils sont des observables intéressants en ce qu'ils témoignent d'un «certain sentiment linguistique ${ }^{26}$.

Dans la lignée de ce plaidoyer pour la prise en compte des analyses non expertes qui affectent le système linguistique, Saussure laisse une place, attendue dans la lignée des acquis néo-grammairiens, aux phénomènes d'analogie mais aussi à des phénomènes plus marginaux, frappés de discrédit, comme ceux de l'étymologie populaire. Le premier cours de linguistique générale livre une analyse approfondie et exempte des préjugés normatifs de son temps (v. Béguelin 1995) de ce type de phénomènes qui sont placées, aux côtés du changement phonétique et de la création analogique comme le troisième facteur -plus marginal- du changement linguistique.

Cet intérêt saussurien pour l'étymologie populaire s'était déjà manifesté peu d'années auparavant dans la correspondance avec Charles Bally (Amacker 1994b,

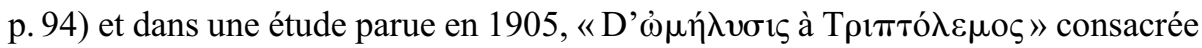
à l'analyse de la formation de deux composés grecs: $\omega \mu \eta ́ \lambda v \sigma ı \varsigma$ qui est issu du

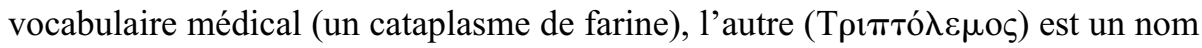
propre. Contre l'étymologie savante admise, qui fait dériver les deuxièmes

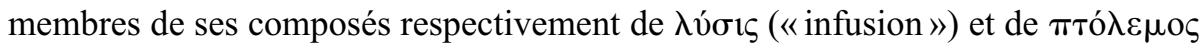
(«guerre »), Saussure voit dans ces deuxièmes membres respectivement des formes

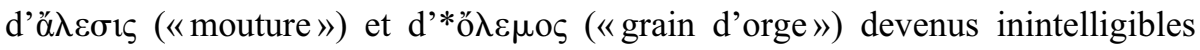
pour les locuteurs. C'est là, écrit-il, un fait «d'étymologie populaire imparfaite, ou "inachevée", où le second terme reste privé de sens, livré à l'inconnu, sans essai d'interprétation" (Saussure [1905] 1922, p. 578). Saussure met en relation, dans cet article, les étymologies savantes des grammairiens et les rapprochements spontanés des locuteurs qui cherchent à remotiver les éléments du système qui leur paraissent obscurs :

Les entreprises de l'étymologie populaire égalent ou dépassent en ingéniosité celles du grammairien, c'est là le facteur à ne pas jamais oublier complètement. On commettrait des erreurs inverses en ce cas. Alors même qu'elle se contente parfois d'un résultat imparfait ( $\omega \mu \eta ́ ~ \lambda v \sigma ı \varsigma)$, les petits miracles qui s'accomplissent ailleurs sous ce principe peuvent émerveiller le linguiste, et le tromper fort bien à chaque instant, de sorte que si le nom de Triptolème n'était que 
l'arrangement d'un composé sans rapport à $\pi \tau o ́ \lambda \varepsilon \mu o \varsigma$ (composé devenu inintelligible par l'oubli du sens d'ỏ $\lambda \varepsilon \mu o \varsigma$ ), le fait pourrait difficilement passer en lui-même pour offrir une particularité bien remarquable. (Ibid., p. 583)

Ce primat accordé aux analyses spontanées des locuteurs et à la conscience des sujets parlants comme observatoire de la langue en synchronie pose aussi en retour la question de la validité des opérations effectuées par les grammairiens qui ne sont pas sanctionnées par la conscience des sujets parlants. C'est tout l'objet du chapitre sur les «entités abstraites» que l'on trouve dans le troisième cours.

\section{L'ABSTRAIT DE LA LANGUE}

La définition d'un état de langue via la conscience des sujets parlants induit une méfiance de principe pour les abstractions linguistiques entendues comme les descriptions non ratifiées par le ressenti des locuteurs. Aussi la notion d'entité abstraite ne reçoit pas de statut théorique dans les deux premiers cours. Dans le premier, Saussure se contente d'interroger les limites de l'«abstraction grammaticale » en se demandant «dans quelle mesure le classement de la langue et du grammairien se correspondent» (CLG/E 2779 I R 2.36). Le deuxième cours dénonce également le «danger des abstractions» en opposant, au sujet de la délimitation des unités linguistiques, «l'abstraction des grammairiens» et le «concret» dont le critère est «ce qui est dans le sentiment des sujets parlants» (CLG/E 1737 II R 42) ${ }^{27}$. Dans ce même cours néanmoins, le problème de la délimitation des unités fait jaillir un sens concurrent d' «abstraction» :

Est-ce que mois singulier et mois pluriel sont le même mot? Alors cheval, chevaux sont aussi le même mot; mais alors pour y trouver une unité il ne faut prendre ni cheval ni chevaux mais ce qui résulte en moyenne des deux : nous faisons une abstraction, nous prenons comme unité quelque chose qui n'est plus donné directement, qui est déjà le résultat de l'opération de l'esprit. (CLG/E 1730 II R 33)

Le problème de la définition des unités confère une nouvelle signification à l'opposition concret/abstrait. Est désignée comme concrète une unité qui correspond à une segmentation de la chaîne du discours et comme «abstraite» une unité qui ne correspond pas à une délimitation du signifiant mais procède d'une opération de l'esprit. Ainsi les mots cheval ou mois sont produits «par abstraction » à partir des unités concrètes attestées dans les discours. Si Saussure conclut que « la langue nous frappe donc $<$ car c'est là son premier caractère $>$ comme ne présentant

27 C'est, une fois de plus, la délimitation des unités, qui soulève le problème de la frontière entre le concret et l'abstrait: "Quand le grammairien vient dire que dans $e k w o-s$ ekwo= radical $<$ cette délimitation $>$ est une abstraction des grammairiens. Et c'est vrai parce que ekwon'était pas ressenti comme une unité par les Latins» (CLG/E 1737 II R 42). 
pas d'unité concrète» et que ce caractère «se résout en un problème » ( $\mathrm{CLG} / \mathrm{E}$ 1752-3 II R 35), il n'aborde pas ce problème des unités abstraites, ni ne pose la correspondance entre les deux emplois qu'il fait du couple concret/abstrait dans le deuxième cours : (1) attribuable aux locuteurs/attribuable aux grammairiens ; (2) segmentable/non segmentable sur le plan de l'expression. La notion d'entité abstraite ne reçoit un traitement théorique que dans le troisième cours. Le chapitre qui lui est consacré figure après celui sur les entités concrètes de la langue; il est introduit en ces termes:

C'est un domaine des plus difficiles à explorer. Nous ne voyons ici que des rayons et non la clarté totale. Il $<$ (ce domaine) $>$ suppose l'étude préalable des entités concrètes. (CLG/E 2165 III C 295-296).

L'embarras du professeur vient de ce que le fondement même de sa démarche linguistique est de se fonder sur les marques morphologiques et de récuser par principe toute opération qui n'aurait pas de correspondance formelle. La notion d'entité abstraite est introduite dans ce cours pour expliquer deux ordres de faits non réductibles aux entités concrètes. D'abord, les faits d'ordre syntagmatique par lesquels Saussure réunit des problèmes morphologiques et syntaxiques ${ }^{28}$. Alors que les morphèmes, les lexèmes ou les constituants syntaxiques sont ce que Saussure appelle des «entités concrètes », leur ordre déterminé par la langue - le troisième cours donne l'exemple de désireux et pas eux-désir ou je dois et pas doisje- relève des «entités abstraites». Cette notion invalide une idée ancienne, relayée notamment par Chomsky (par ex. [1967] 1968, p. 37), selon laquelle la théorie saussurienne de la langue ferait l'impasse sur la syntaxe, reléguée dans le domaine de la parole. La notion d'entité abstraite prouve qu'il n'en est rien même si Saussure affirme ailleurs que la syntaxe est précisément l'endroit d'un «flottement» pour la distinction langue/parole ${ }^{29}$. La structure d'un syntagme, qui va donc du mot à la phrase, est abstraite par les sujets parlants à partir des entités concrètes $^{30}$. Comme l'écrit René Amacker qui a développé, dans Linguistique saussurienne, à partir de la notion d'entité abstraite une syntaxe d'inspiration saussurienne: "l'entité abstraite syntaxique est définie comme étant en rapport dialectique avec les unités dont elle se dégage et qu'elle sert tout à la fois à former»; en cela « elle est une unité de la langue au même titre que les autres » (Amacker 1975, p. 194).

28 Saussure parle de la «syntaxe intérieure des mots » (CLG/E 2538 I R 2.92) et il indique que «les faits de syntaxe tombent dans 〈la〉 syntagmatique» (CLG/E 2158 II R 97).

29 Cf. CLE/E 2022 III C 277. Sur la syntaxe chez Saussure, v. les études récentes d'Arrivé ([2005-2006] 2016) et d'Hagège (2016) qui, curieusement, ne mentionnent pas l'étude la plus aboutie sur la question: Amacker 1975.

30 Une première esquisse de cette idée est formulée dans le premier cours: "Cet ordre [syntaxique], la langue l'abstrait par l'analyse aussi bien que les unités elles-mêmes » (CLG/E 1985 I R 2.33). 
Les entités abstraites interviennent, en second lieu, pour expliquer des faits associatifs, et spécifiquement les paradigmes au sens étroitement grammatical:

En latin : domini, regis, regum il n'y a rien dans le $i$, le is, le um qui coïncide et dont on puisse dire que c'est la même unité et sous-unité. Et cependant il y a ici avec ce support matériel divers, quelque chose qui est la conscience d'une certaine valeur, qui est la même $<$ et dicte un emploi identique. Ici nous quittons tout à fait contact avec support matériel $>$ Il y a une abstraction positive opérée sans doute par tous les sujets parlants. Peut-on méconnaître la puissance présente au sujet parlant, de la valeur de génitif? Cela $<$ des identités de ce genre $>$ peut rentrer aussi dans la notion de procédé. (CLG/E 2177-78-83 III C 297).

Les désinences is, $i$, um forment une identité, alors qu'elles ne sont pas phonologiquement identiques du fait de l'association paradigmatique - au sens spécifique où Saussure utilise le terme de paradigme c'est-à-dire pour désigner les cas de flexion nominale et verbale - opérée par les sujets parlants. L'entité abstraite est donc produite par la reconnaissance d'une relation d'identité. Cette unité est dite «abstraite» parce qu'elle n'a pas de correspondance homogène sur le plan de l'expression mais, compte tenu de sa méfiance pour l' «abstraction», Saussure la tempère immédiatement en la qualifiant «d'abstraction positive sans doute opérée par tous les sujets parlants ». En d'autres termes, l'abstraction au sens (2) est positive car elle est concrète au sens (1).

Ce questionnement autour des paradigmes grammaticaux établis par les grammairiens et de leur réalité pour la conscience des sujets parlants est un questionnement ancien. On le trouve formulé dans d'autres textes, toujours avec l'exemple du génitif. Par exemple, vingt ans plus tôt, dans les manuscrits de l'Essence double:

Une catégorie grammaticale, comme la catégorie du génitif, par exemple, est une chose complètement insaisissable, un mot véritablement destitué de sens dans l'emploi que nous en faisons journellement. Nous ne voulons pas dire, ce qui est certain d'emblée, que cette catégorie n'est ni nécessaire pour l'esprit, ni représentée avec nécessité dans les différentes langues qu’on examinera, ni une dans ce qu'elle embrasse en général, ou en particulier dans telle langue. Nous voulons dire que dans une langue déterminée, où existe un "génitif», on ne sait jamais ce qui est entendu de moment en moment, de page en page, de ligne en ligne, par ce mot «génitif» ou ce qu'on veut exactement généraliser en parlant de la catégorie du génitif dont jouit la grammaire de cette langue. Prenons par exemple le génitif grec [

(Saussure 2011, p. $185=$ Saussure 2002, p. 55)

Puis de nouveau dans le deuxième cours :

La déclinaison est certainement une des manières dont les formes sont groupées pour le sentiment des sujets parlants. C'est la liaison des divisions que l'on pose qui manque. Une fois explorées il est possible qu'elles aient à changer. La méthode est simplement d'observer, de considérer comme réel ce que la 
conscience de la langue reconnaît, ratifie, et comme irréel ce qu'elle ne reconnaît pas. Cela met la méthode à la portée de tous: consiste dans l'observation intérieure rectifiée par l'observation de tous. Par exemple un tableau de déclinaison est-il une invention de grammairien=existe-t-il ?» (CLG/E 2163 II $\mathrm{R}$ 108)

Le troisième cours apporte une réponse à cette dernière question en faisant converger les deux acceptions du couple concret/abstrait. Les tableaux de déclinaison conçus par les grammairiens «existent», c'est-à-dire qu'ils ont une existence pour la conscience des sujets (concret au sens 1) mais ils sont des entités abstraites (au sens 2). Et Saussure de préciser la signification du couple concret/ abstrait dans la définition des unités abstraites de la langue:

Il y a un sens où l'on pourrait dire au contraire que rien ne peut être abstrait dans la langue; on pourrait justifier cette terminologie en disant: dans la langue est concret tout ce qui est présent à la conscience des sujets parlants ; en considérant comme abstraite telle ou telle distinction n'appartenant qu'aux grammairiens, mais non ratifiée par la conscience des sujets parlants. Ce n'est pas dans ce sens que nous avons pris concret et abstrait.

Nous avons réservé le terme de concret le cas où l'idée a directement son appui dans une unité sonore. Abstrait ayant indirectement son appui dans une opération des sujets parlants. (CLG/E 2195 III C 298-299).

Cette définition de l'abstrait de la langue qui, par-delà l'absence de marques morphologiques homogènes trouve son existence dans l'opération des sujets parlants apparaît comme l'aboutissement d'un long cheminement. Et notamment ce passage du troisième cours qui définit les «entités abstraites » à partir du génitif et des paradigmes fait écho à un passage très commenté des manuscrits de l'Essence double (Saussure 2002, p. 87-88=Saussure 2011, p. 186-189) où Saussure développe, à partir du même exemple du génitif, le concept de «postméditation », c'est-à-dire d'opération non consciente attribuable au locuteur pour transformer les oppositions entre des entités purement négatives en entités positives pour s'approprier la langue ${ }^{31}$. Dans les deux cas, c'est le rapport entre système linguistique et cognition qui est posé et, dans les deux cas, les opérations non nécessairement conscientes que Saussure attribue aux locuteurs sont des aprèscoups, en aucun cas des préalables, qui permettent d'organiser le jeu des oppositions et des différences en des entités illusoirement positives, en séries, en associations.

Les «opérations de l'esprit», aussi appelées "procédés», jouent un rôle important dans la théorisation saussurienne : elles sont le processus par lesquels les sujets parlants appréhendent la négativité du système linguistique. Dans ce

31 Sur ce concept de «post-méditation» ou «de post-élaboration», v. Gambarara, Russo Cardona, Fadda, Chidichimo (2008). 
processus, la cognition intervient en amont du système. Du concept de «postméditation» esquissé dans l'Essence double à la formule «il n'y a pas de préméditation » qu'on trouve dans le premier cours de linguistique générale au sujet du langage intérieur ${ }^{32}$, la réflexion saussurienne dessine une conception de la langue qui ne correspond, eu égard au rôle que l'activité cognitive des sujets parlants joue dans son fonctionnement, ni à l'immanentisme qu'y a vu une certaine lecture structuraliste ni aux tentatives actuelles d'assimilation de certaines lectures cognitivistes.

\section{BiBLIOGRAPHIE}

\section{Sources primaires}

Bally, Charles, 1909. Traité de stylistique française, Genève, Georg.

Henry, Victor, 1896. Antinomies linguistiques, Paris, Alcan.

Naville, Adrien, 1901. Nouvelle classification des sciences, étude philosophique, Paris, Alcan.

Saussure, Ferdinand de, 1922. Recueil des publications scientifiques de Ferdinand de Saussure, éd. Charles Bally \& Léopold Gautier, Lausanne: Payot.

- [1916] 1967. Cours de linguistique générale, publié par Charles Bally et Albert Sechehaye, éd. Tullio de Mauro, Paris, Payot.

- 1968. Cours de linguistique générale. Tome 1, éd. critique, Rudolf Engler, Wiesbaden, Otto Harrassowitz.

- 1974. Cours de linguistique générale, Tome 2 : Appendice. Notes de F. de Saussure sur la linguistique générale, éd. critique, Rudolf Engler, Wiesbaden Otta Harrassowitz.

- 2002. Écrits de linguistique générale, éd. Simon Bouquet \& Rudolf Engler, Paris, Gallimard.

- 2011. Science du langage, éd. René Amacker, Genève, Droz.

Sechehaye, Albert, 1908. Programme et méthodes de la linguistique théorique. Psychologie du langage, Paris, Champion.

Wundt, Wilhelm, 1900. Die Völkerpsychologie I. Die Sprache, Leipzig, Engelmann.

\section{Sources secondaires}

Amacker, René, 1975. Linguistique saussurienne, Genève, Droz.

- 1994a. «La théorie linguistique de Saussure et la psychologie », Cahiers Ferdinand de Saussure 48, 3-13.

- 1994b. «Correspondance Bally-Saussure », Cahiers Ferdinand de Saussure 48, 95-127.

Arrivé, Michel, [2012] 2016. «Conscience de la langue et inconscient chez Ferdinand de Saussure », Saussure retrouvé, Paris, Garnier, 77-97.

— [2005-2006] 2016. «La syntaxe chez Saussure », Saussure retrouvé, Paris, Garnier, 99113.

Béguelin, Marie-José, 1995. «Saussure et l'étymologie populaire», Linx 7, 121-138: https://doi.org/10.4000/linx.1131

Bergounioux, Gabriel, 1995. «Saussure ou la pensée comme représentation », Linx 7, 173186: https://doi.org/10.4000/linx.1145

Chidichimo, Alessandro, 2009. «Saussure et o sentimento: a forma do sentimento linguistico », RUA 15, 108-123: http://www.labeurb.unicamp.br/rua/

- 2017. "Une source du premier cours de linguistique générale de Saussure, octobre $1906 »$, Semiotica 217, 195-213 : https://doi.org/10.1515/sem-2016-0073 
Chiss, Jean-Louis et Puech, Christian, 1983. «La linguistique et la question de l'écriture : Enjeux et débats autour de Saussure et des problématiques structurales », Langue Française 59, 5-24.

Chomsky, Noam, [1967] 1968, Le langage et la pensée, trad. Louis-Jean Calvet, Paris, Payot.

De Palo, Marina, [2001] 2016. L'invention de la sémantique. Bréal et Saussure. Trad. Anna Maria Perrone, Limoges, Lambert-Lucas.

Fadda, Emanuele, 2013. «Sentiment : entre mot et terme. Quelques notes sur le travail et la langue de Ferdinand de Saussure », Cahiers Ferdinand de Saussure 66, 49-65.

Gambarara, Daniele, Russo, Cardona, Toma, Fadda, Emanuele, Chidichimo, Alessandro, 2008. «Système et cognition. Quaternions et parallélie dans "De l'Essence double du langage" ", Cahiers Ferdinand de Saussure 61, p. 77-129.

Godel Robert, 1957. Les sources manuscrites du Cours de linguistique général de F. de Saussure, Genève, Droz.

Hagège, Claude, 2016. "À propos de la vision saussurienne de la syntaxe», Recherches Sémiotiques / Semiotic Inquiry 34, 97-112.

Joseph, John E, 2012. Saussure, Oxford, Oxford University Press.

Koerner, Konrad, 1971. Ferdinand de Saussure. Origin and development of his linguistic theory in western studies of language, thèse de doctorat, Simon Fraser University.

Morpurgo-Davies, Anna, 1978. "Analogy, segmentation and the early Neogrammarians », Transactions of the Philological Society 76, 36-60.

Samain, Didier, 2016. "Portrait du linguiste en jeune grammairien», Recherches Sémiotiques / Semiotic Inquiry 34, 137-156.

Testenoire, Pierre-Yves, 2016. «Ce que les théories du discours doivent à Saussure : note sur la note dite "sur le discours" », Cruz, Marcio Alexandre, Piovezani, Carlos et Testenoire, Pierre-Yves (éd.), Le discours et le texte: Saussure en héritage, Academia-Bruylant, Louvain-la-Neuve, 109-132.

— 2017. «La langue est une institution SANS ANALOGUE (si l'on y joint l'écriture)»: l'écriture comme problèmes dans la réflexion théorique de Saussure », Semiotica 217, 117-133 : https://doi.org/10.1515/sem-2016-0061

- 2018. «Jeu de mot, jeu phonique et anagramme dans la réflexion linguistique de Saussure», Lecolle, Michèle et Full, Bettina (éd.), Jeux de mots et créativité linguistique, Berlin, De Gruyter, à paraître.

Vincent, François, 2013. Le premier cours de linguistique générale professé par Ferdinand de Saussure à Genève $(\mathrm{ClCa} / \mathrm{FV})$ Cours I et Sténographie Caille-Transcription et commentaires, thèse de doctorat, Université Paris-Est Créteil Val de Marne. 